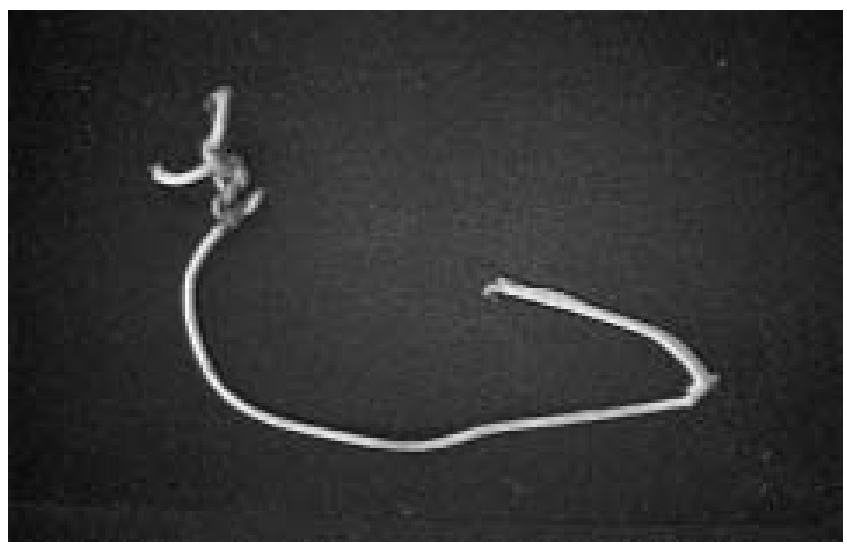

Figure 1. Low magnified image of broken PDS suture $(\times 5)$. No loose knot was observed. Broken sites were $3 \mathrm{~mm}$ apart from the knot. Another abrasion injury was observed $20 \mathrm{~mm}$ away from the broken site. One is the part of the cord just above the sternum, and the other is the part just below the sternum.

weeks and $30-40 \%$ at 6 weeks after implantation. This is another reason for broken sutures.

Broken sutures made of PDS cord may occur several weeks after surgery when patients have a persistent cough. PDS cord sutures do not have sufficient reliability to close the sternum, especially in patients with chronic obstructive pulmonary dysfunction and obesity. ${ }^{1}$

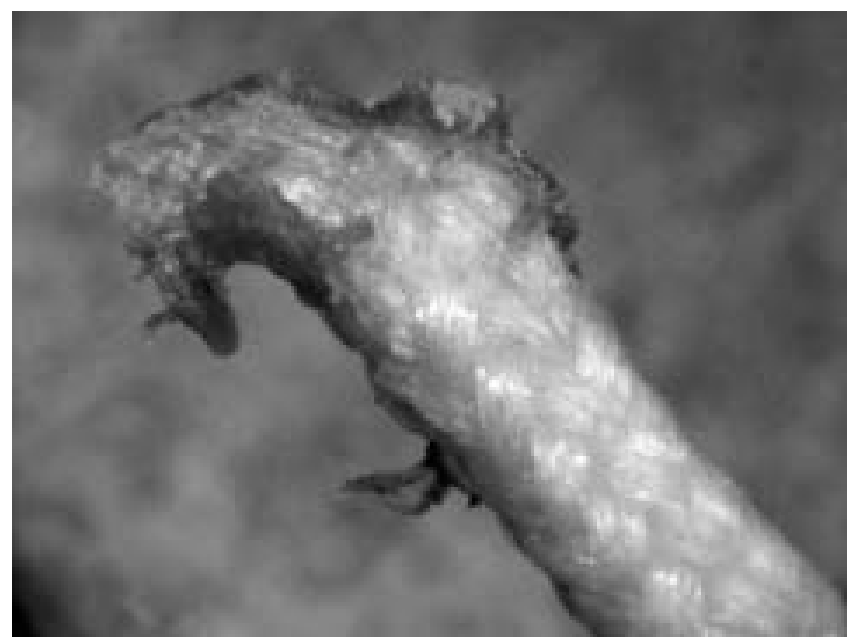

Figure 2. Magnified image of site of break (a) $(\times 60)$. The cord stump gradually became thin, kinked with curled filaments, and had possibly been torn off.

\section{Reference}

1. Ray JA, Doddi N, Regula D, Williams JA, Melverger, Polydioxane (PDS), a novel monofilament synthetic absorbable suture. Surgery, Gynecology \& Obstetrics 1981;153:497-507.

\title{
Intraoperative finding of structural abnormality of bovine pericardial aortic prosthesis
}

Uberto Da Col, MD, Davide Di Lazzaro, MD, Gino Di Manici, MD, Isidoro Di Bella, MD, and Temistocle Ragni, MD

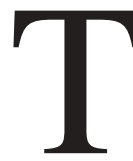

he bovine pericardial valve is an excellent valve, with a long, widespread use without reported intraoperative failures. ${ }^{1}$ However, 4 intraoperative structural failures of bovine pericardial bioprostheses placed in the mitral position were recently reported. ${ }^{2}$ The authors of this report hy-

From the S.C. di Cardiochirurgia, Azienda Ospedaliera di Perugia, Ospedale R. Silvestrini, Perugia, Italy.

Received for publication Nov 29, 2005; revisions received Dec 15, 2005; accepted for publication Dec 22, 2005.

Address for reprints: Davide Di Lazzaro, S.C. di Cardiochirurgia, Ospedale R. Silvestrini, S. Andrea delle fratte 06156 Perugia, Italy (E-mail: davide.dilazzaro@ospedale.perugia.it).

J Thorac Cardiovasc Surg 2006;131:1175-6

$0022-5223 / \$ 32.00$

Copyright $\odot 2006$ by The American Association for Thoracic Surgery doi:10.1016/j.jtcvs.2005.12.044 pothesized that the failures were caused by the distortion of the normal planar geometry of the prosthesis caused by mitral annular disease; this leads to failure of adequate central leaflet position and coaptation.

We report the intraoperative finding of a structural abnormality of the same type of bovine pericardial valve, detected after implantation in the aortic position, before closure of the aorta.

\section{Clinical Case}

A 69-year-old man underwent elective operation for aortic valve stenosis and dilatation of the ascending aorta. His heart had a poor global functionality, with an echocardiographic ejection fraction of $20 \%$ to $25 \%$. The operative approach was through median sternotomy; the ascending aorta appeared dilatated starting from the sinotubular junction up to $1.5 \mathrm{~cm}$ before the origin of the innominate artery. The Valsalva sinus and aortic arch appeared slightly dilated, and cardiomegaly (mainly for dilatation of the left ventricle) was observed. We opted for separate replacement of the aortic 


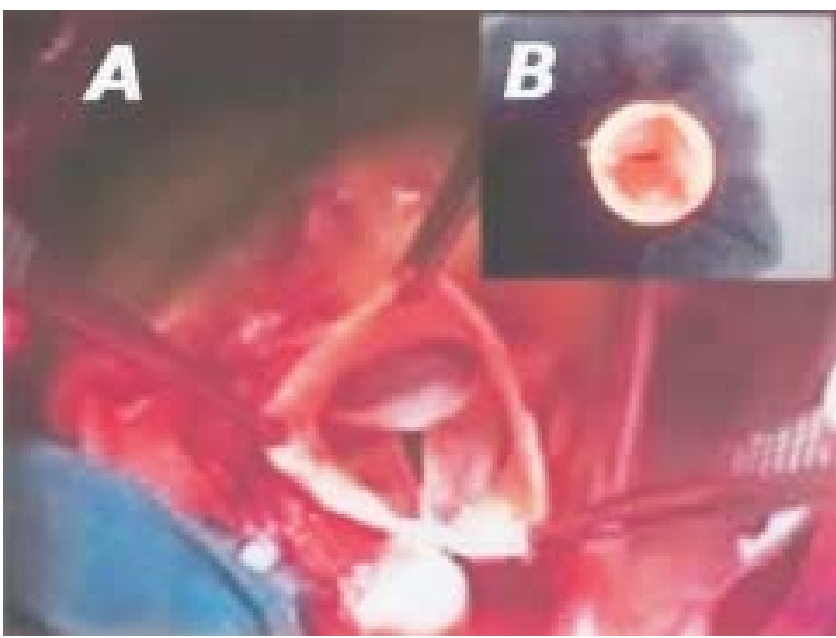

Figure 1. A, The aortic prosthesis in situ: Area of separation between commissures is clearly visible. $B$, The prosthesis removed: "Spear's blade" sign is still visible.

valve and ascending aorta. Standard aortic arch and single atrial cannulation were used, along with selective cardioplegia into the coronary ostia. After removal of a bicuspid, severely calcified aortic valve, a 27-mm Edwards Perimount stented pericardial aortic prosthesis (Edwards Lifesciences, Irvine, Calif) was implanted with subannular 2-0 Tycron interrupted pledgeted sutures. After removing the valve holder, we noted the coaptation edge of the 3 commissures was not normally adapted in the diastolic position, but showed an area of separation shaped as a "spear's blade" (Figure 1, A).

The annulus inspection showed no distortion, and the valve stents appeared normal with no sign of dislocation; neither the Valsalva sinus nor the left ventricle outflow tract showed any obstacle to the normal motion of the valve's cusps (Figure 2).

An attempt to manually approximate the 2 distant cusps produced only further distortion of the remaining cusp. Finally, a hydrodynamic test was performed. Saline solution injected into the aortic bulb with vent-forced aspiration failed, indicating severe prosthetic incompetence. Because of the doubts raised by these findings concerning the competence of the prosthetic valve and its durability, we decided on re-replacement, inserting a new prosthesis of the same type and size as the previous one. The new prosthesis showed no structural anomaly. Even after explantation, the old one showed the same deficit in cusp coaptation (Figure 1, $B)$. The operation was completed without any problem, and the postoperative course was uneventful except that a pacemaker implantation was necessary because of postoperative complete arteriovenous block.

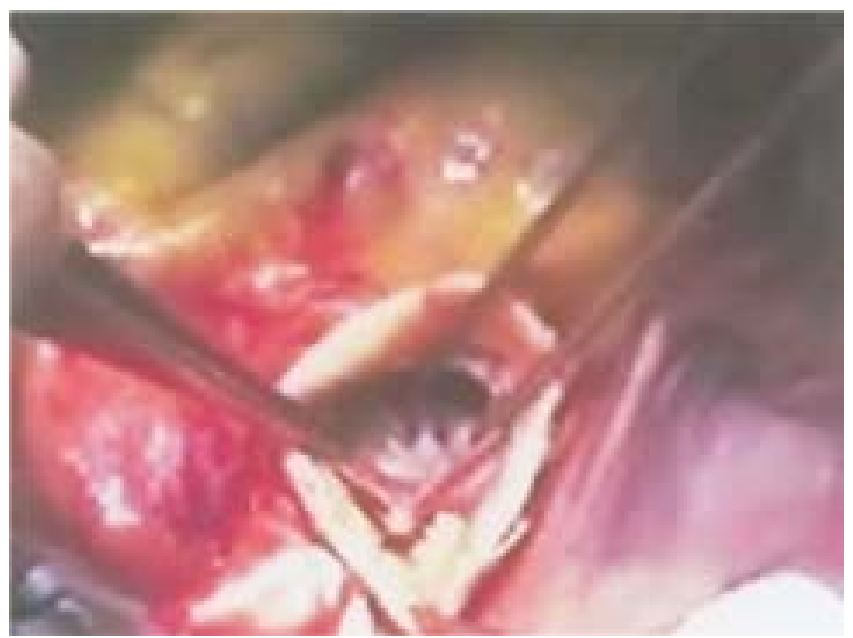

Figure 2. After removal of the prosthesis, inspection of the left ventricle outflow tract shows no obstacle to the cusps' motion.

\section{Comment}

In the recent report ${ }^{2}$ on the failure of 4 bovine pericardial valves, the authors hypothesized that the failures were caused by distortion of normal planar geometry of the prosthesis by a diseased mitral annulus. In this report, we suspect an intrinsic structural defect, not caused by some external factor, either iatrogenic (ie, distortion caused by sutures) or anatomic, because nothing was found on inspection of the prosthesis and cardiac structures. The aforementioned defect remained even after the prosthesis was removed, indicating structural failure. We were able to note the defect only after removal of the valve holder, because its position does not allow an easy inspection of the valve unless done specifically. Moreover, a second prosthesis was implanted (identical in size, type, and technique of suturing) without any sign of cusp distortion. It is noteworthy that although the position differed (mitral vs aortic), relatively large implanted prostheses $(27-29 \mathrm{~mm})$ were used in our case and the previously reported cases. ${ }^{1,2}$

These experiences should alert surgeons to the occasional structural failure of such a prosthesis.

\section{References}

1. Marchand M, Aupart M, Norton R, Goldsmith IRA, Pelletier C, Pellerin M, et al. Twelve-year experience with Carpentier-Edwards PERIMOUNT pericardial valve in the mitral position: a multicenter study. J Heart Valve Dis. 1998;7:292-8.

2. Saunders PC, Grossi EA, Esposito RA, Bizekis CS, Strong MD, Colvin SB. Failure of four bovine pericardial mitral prostheses. $J$ Thorac Cardiovasc Surg. 2004;127:267-8. 